Check for updates

1 Clinical Ethics and Law, Faculty of Medicine, University of Southampton, Southampton, UK

2 Wessex Clinical Genetics Service, Princess Anne Hospital, Southampton, UK

3 Department of Public Health and Primary Care, Department of Oncology, Cambridge Cancer Centre, University of Cambridge, UK

4 Yorkshire Regional Genetics Service, Leeds, UK

5 Shipley Medical Practice, Affinity Care, Shipley, UK

6 Wellcome Centre for Human Genetics, University of Oxford, Oxford, UK

Correspondence to A Lucassen anneke.lucassen@well.ox.ac.uk Cite this as: BMJ 2021;375:n2376 http://dx.doi.org/10.1136/bmj.n2376 Published: 14 October 2021

PRACTICE POINTER

\title{
Care of men with cancer-predisposing BRCA variants
}

\author{
Rachel Horton, ${ }^{1,2}$ Paul Pharoah, ${ }^{3}$ Judith Hayward, ${ }^{4,5}$ Anneke Lucassen ${ }^{1,6}$
}

\section{What you need to know}

- Men and women are equally likely to inherit or pass on a cancer-predisposing $B R C A$ variant-family history of cancers needs to encompass both sides of the family

- Men with cancer-predisposing BRCA variants have an increased risk of developing breast cancer and are advised to be breast aware

- Men with cancer-predisposing BRCA2 variants have an increased risk of developing aggressive prostate cancer (men with cancer-predisposing BRCA1 variants may also have an increased risk); it is not yet known whether prostate specific antigen screening reduces mortality in men with cancer-predisposing $B R C A$ variants

- The European Association of Urology recommends that PSA screening is offered to men with cancer-predisposing $B R C A 2$ variants from 40 years of age after discussion of the risks and benefits

Around one in 260 men $(\sim 0.4 \%)$ inherits a cancer-predisposing $B R C A$ variant that increases their risk of developing prostate, pancreatic, and breast cancer and may affect the health of their family. ${ }^{12}$ Most of these men are currently unaware that they have a cancer-predisposing $B R C A$ variant, but as genetic testing becomes more common, more men will need medical advice about what having such a variant means for them and their families.

Men are just as likely as women to have a cancer-predisposing BRCA variant, but many people perceive these variants as only being relevant to women. Paradoxically, this could lead to women at very high risk of breast and ovarian cancer missing out on screening and risk-lowering treatment despite a concerning paternal family history. Clinicians might also be less attuned to paternal family history of cancer in assessing women's breast cancer risk. ${ }^{3}$ This practice pointer covers what cancer-predisposing $B R C A$ variants are, who might be tested; and what health issues men and their clinicians need to know about. We refer to men, but the article also applies to transwomen and some non-binary people.

\section{What are cancer-predisposing $B R C A$ variants?}

$B R C A 1$ and $B R C A 2$ are tumour suppressor genes that code for DNA repair proteins. Certain variants in these genes predispose to cancer (primarily breast, ovarian, prostate, and pancreatic, and for BRCA2 possibly melanoma $^{4}$ ) (fig 1). The predisposition to cancer is inherited in an autosomal dominant way, ie, each and every time a person with a cancer-predisposing $B R C A$ variant has a child, they have a 1 in 2 or 50:50 chance of passing their BRCA variant on to the child. This is regardless of whether parent or child is male or female.

Many men and some women with cancer-predisposing $B R C A$ variants will never develop an associated cancer (fig 1 ). The cancer risks associated with cancer-predisposing $B R C A$ variants are modified by lifestyle factors and other inherited genetic variants. Polygenic risk scores (calculated by looking at multiple common genetic variants across the genome, each with a tiny individual effect) show potential to refine cancer risk predictions for people with cancer-predisposing $B R C A$ variants, but cannot remove uncertainty as to whether a given patient will or will not develop cancer. ${ }^{10}$ 

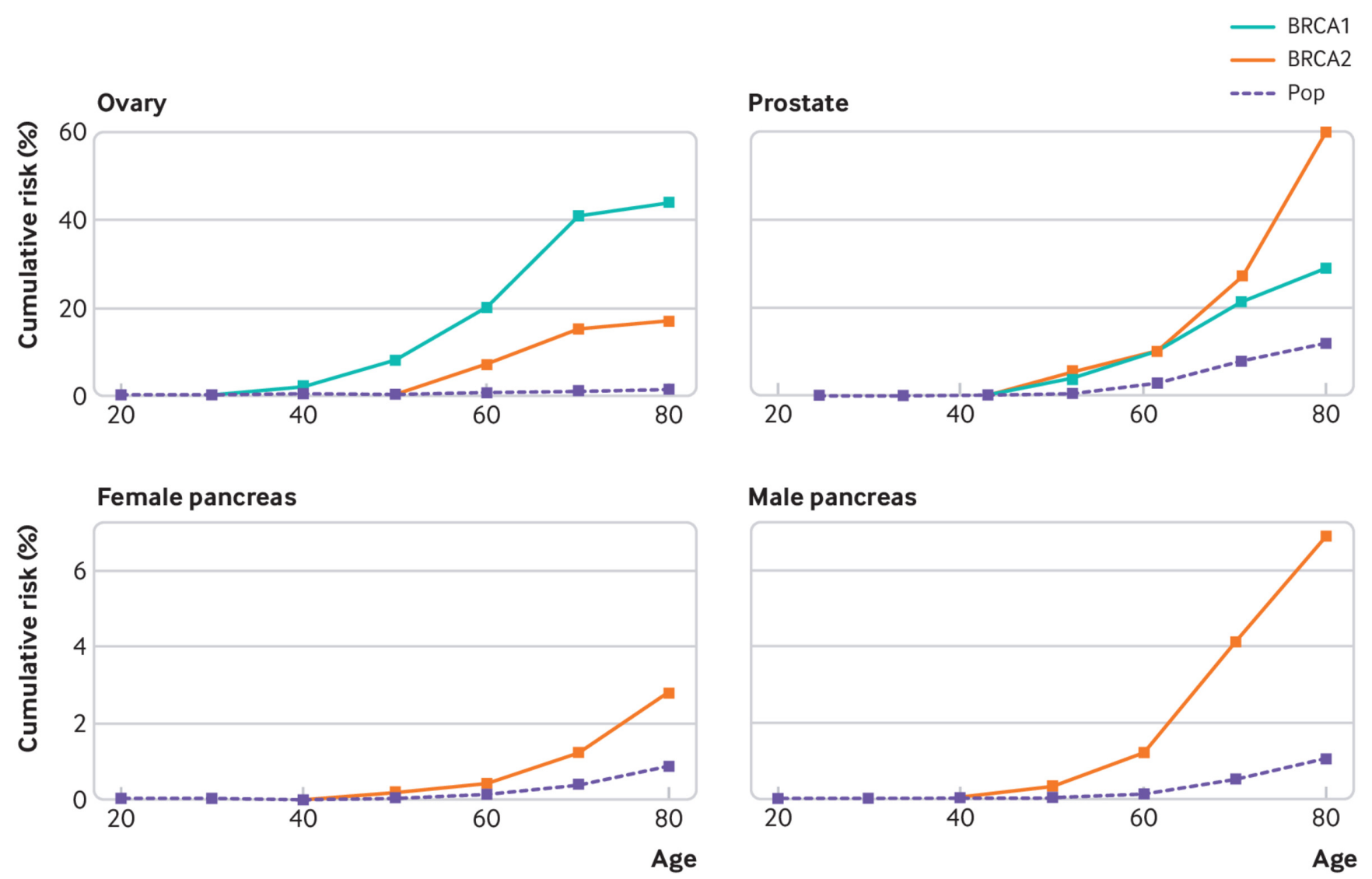

Fig 1(a) | Risk of ovary, prostate, and pancreatic cancer associated with cancer-predisposing BRCA variants (cumulative cancer risks are shown on different scales). Cancer-predisposing BRCA1 variants increase the risk of pancreatic cancer (relative risk 4.11$)^{5}$ but data are emerging, and cumulative risk figures are not readily available. Data on ovary cancer from Kuchenbaecker et al 20176; prostate from Nyberg et al 2020, ${ }^{7}$ and pancreatic cancer from van Asperen et al $2005 .{ }^{8}$ Population data are for England and Wales 2016 (Office for National Statistics)
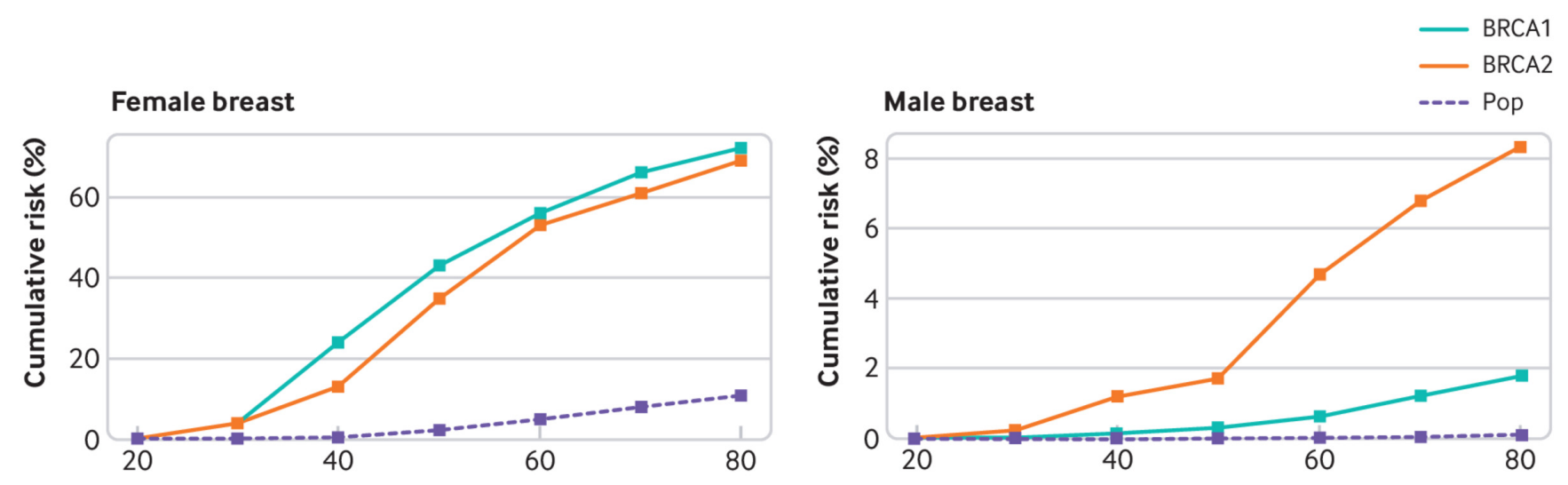

Fig 1(b) | Risk of female and male breast cancer associated with cancer-predisposing BRCA variants. Data from Tai et al $2007^{9}$ Population data are for England and Wales 2016 (Office for National Statistics)

\section{Describing genetic variants}

Various terms are used to describe genetic variants that increase risk of disease. As with many medical terms, different people might understand different things by the same term.

The term "mutation" is often used in patient leaflets, patient support forums, and in UK school curriculums. However, some find the term offensive ${ }^{36}$ and it is technically incorrect since it suggests the mutation has happened in that person. "Faulty gene" is also often used in patient resources, and a 2007 Australian survey indicated that this term was most preferred by the general community and by patients recruited via cancer genetics clinics. 37

"Pathogenic" or "likely pathogenic variant" is commonly used in laboratory genetic reports. People are increasingly familiar with the term "variant" in the context of covid-19, but "pathogenic" may be less well 
understood by people without a healthcare background, so

"disease-causing" is sometimes used instead. However, this in turn may give the false impression that everyone with a "disease-causing $B R C A$ variant" will inevitably develop an associated cancer. Other terms in common use include: "disease-risk variant," "genetic risk factor," "disease-predisposing variant," "loss-of-function variant," "gene alteration," "gene change" and for people with cancer-predisposing $B R C A$ variants, "BRCA carriers." Importantly there are also many variants in $B R C A_{1}$ and $B R C A 2$ which are not associated with cancer risks.

In discussion with our hospital Patient and Public Involvement group it was evident that different people found different terms most helpful. We use the term "cancer-predisposing variant" here as it is informative and technically accurate, but whatever term you use to describe cancer-predisposing $B R C A$ variants, key messages to convey are

- variants are very likely to be inherited rather than to have happened for the first time in the tested person

- variants are risk factors for particular cancers rather than certainties that they will develop.

Thousands of different variants within the BRCA1 and BRCA2 genes have been described, and classifying which are benign and which increase cancer risk can be challenging. ${ }^{11}$ As more data are gathered, classifications may shift. For example a Canadian laboratory examined the $B R C A$ variants they identified over a five year period and found that $12 \%$ were reclassified $(75 \%$ of these were downgraded-ie, they are now thought to be less likely to predispose to cancer than they were when the $B R C A$ test was originally done). ${ }^{12}$ Diagnostic tests involve sequencing of BRCA1 and BRCA2 (and perhaps other genes, eg, $P A L B 2$ ), aiming to detect any variant present that might increase cancer risk.

\section{How would a man find out that he has a cancer-predisposing $B R C A$ variant?}

In the UK, diagnostic BRCA testing is currently offered to people with a $10 \%$ or greater chance of having a cancer-predisposing $B R C A$ variant. ${ }^{13}$ Most diagnostic $B R C A$ tests are done for women, but some men will find out their $B R C A$ status via this route, as men with breast cancer at any age are eligible to be tested, ${ }^{14}$ and some men with prostate or pancreatic cancer may have testing to determine eligibility for poly(ADP-ribose) polymerase (PARP) inhibitors (often in the context of clinical trials).

More commonly, men are offered targeted BRCA testing after a cancer-predisposing $B R C A$ variant is identified in their family. Usually this will be predictive testing (ie, the man has no personal medical history suggestive of a cancer-predisposing BRCA variant), although occasionally it may be explanatory (eg, in men known to have prostate cancer). The laboratory would need details of the specific cancer-predisposing BRCA variant in the man's family in order to test for it (eg, a genetic report from an affected family member) and such targeted testing would not detect any other cancer-predisposing variants.

Around $1.5 \%$ of patients with prostate cancer have a cancer-predisposing $B R C A$ variant, ${ }^{15}$ but a personal medical history of prostate cancer is not an indication for diagnostic $B R C A$ testing in the UK. However, for patients with younger-onset, aggressive disease, a thorough family history can explore the possibility of a familial cancer-predisposing BRCA variant. A family history that includes, for example, bilateral breast cancer, male breast cancer, or multiple people affected by breast or ovarian cancer (especially at younger ages), probably warrants discussion with your local genetics service.
Some men might access BRCA testing outside standard clinical pathways, such as through direct-to-consumer genetic testing or research studies. ${ }^{16}$ These tests are of variable quality and scope, and further scrutiny may be needed to confirm that the variant is really present or really represents a risk. ${ }^{17}$

\section{Case studies}

\section{Cancer-predisposing $B R C A$ variants increase the risk of aggressive prostate cancer}

Rahul is a 40 year old man who had a test via a genetics clinic for the cancer-predisposing BRCA2 variant identified in his aunt. Rahul was also found to have the BRCA2 variant and is concerned about developing prostate cancer. He books an appointment with you to discuss prostate specific antigen (PSA) screening.

Men with cancer-predisposing BRCA2 variants like Rahul have an increased risk of prostate cancer: a recent meta-analysis indicated an odds ratio of $2.64,{ }^{15}$ and a large prospective cohort study in the UK and Ireland found a $27 \%$ absolute risk of developing prostate cancer by age 75 , rising to $60 \%$ by age $85^{7}$ (for comparison, population risk by age 85 is $16 \%$ in England and Wales based on Office for National Statistics 2016 data). When prostate cancer occurs in a man with a cancer-predisposing $B R C A 2$ variant, it tends to be more aggressive. ${ }^{18}$ The evidence has been inconsistent regarding the impact of cancer-predisposing $B R C A 1$ variants on prostate cancer but a subtler effect is probable, with an odds ratio of $1.35,{ }^{15}$ and the relative risk increase is higher at younger ages. ${ }^{19} 20$

The IMPACT study is an ongoing international prospective cohort study of more than 3000 men to examine the use of PSA screening in men with cancer-predisposing $B R C A$ variants. Based on interim results, the researchers recommend that men with cancer-predisposing BRCA2 variants are offered systematic PSA screening, ${ }^{21}$ and this could be considered for Rahul. This is because after three years of PSA screening, men with cancer-predisposing $B R C A 2$ variants proved to have a higher incidence of prostate cancer, were younger at diagnosis, and were more likely to have clinically significant tumours. The positive predictive value of a PSA >3.0 $\mathrm{ng} / \mathrm{mL}$ was higher in men with cancer-predisposing $B R C A 2$ variants than in controls ( $31 \%$ versus $18 \%$ ).

Discuss with Rahul the pros and cons of PSA screening and be clear about the current limits of medical knowledge. It would be appropriate to include in this discussion that if a prostate cancer does develop in a man with a cancer-predisposing BRCA2 variant, it is more likely to be clinically significant. Interim analysis from the IMPACT study shows that after four screening rounds (annual PSA) for men aged 55-69 with cancer-predisposing BRCA2 variants, you would expect to detect one clinically significant prostate cancer for every 13 men screened..$^{21}$ The European Association of Urology recommends offering PSA based prostate cancer screening to men with cancer-predisposing $B R C A 2$ variants who have been counselled on the potential risks and benefits of screening from the age of 40 although they do not specify a screening interval. ${ }^{22}$ It is not yet known whether PSA screening will reduce mortality from prostate cancer in men with cancer-predisposing BRCA2 variants.

Research is ongoing as to the role of PSA screening for men with cancer-predisposing $B R C A 1$ variants: an interim analysis found no differences in age or tumour characteristics between men with cancer-predisposing BRCA1 variants and controls. ${ }^{21}$ 


\section{Breast awareness is important for men with cancer-predisposing} $B R C A$ variants

Jakob is a 50 year old man who books an appointment to discuss a painless "cyst" near his left nipple that he noticed several months ago. On examination, you notice that he has an inverted nipple and ipsilateralaxillary lymphadenopathy. When you ask about his family history, he tells you that his father died of prostate cancer in his 60 .

Men with cancer-predisposing BRCA variants have an increased lifetime risk of developing breast cancer: $8.3 \%$ for $B R C A 2$ and $1.8 \%$ for $B R C A 1$, compared with $0.1 \%$ in the general population. ${ }^{9}$ As in women, breast cancer in men most commonly presents as a painless mass. Although nipple involvement tends to be seen earlier due to the smaller amount of breast tissue, ${ }^{23}$ male breast cancer is often diagnosed at an advanced stage. ${ }^{23}$ Men with cancer-predisposing $B R C A$ variants are at higher risk of developing breast cancer, but any man with symptoms of breast cancer warrants urgent referral to a breast clinic in the same way women presenting with concerning symptoms would be referred, regardless of BRCA status.

Evidence regarding breast cancer characteristics in men with cancer-predisposing BRCA variants is limited. Two studies analysing tumour grading, staging, and receptor status in men with breast cancer suggest that cancer-predisposing BRCA2 variants are associated with more aggressive cancers. ${ }^{24} 25$

Evidence is also lacking on breast cancer screening in men with cancer-predisposing $B R C A$ variants, and practice varies. ${ }^{26}$ In the $\mathrm{UK}$, men with cancer-predisposing $B R C A$ variants are advised to be breast aware, ie, to know how their breasts usually look and feel, and seek medical advice if they notice changes or have any concerns. ${ }^{13}$ Men might be more likely to delay seeking care for a new breast lump, perhaps waiting until the lump becomes painful or changes the overlying skin. A study in Hong Kong of men with breast cancer found that median duration from symptoms to first medical consultation was 12.4 months, and $84 \%$ were not aware (before their diagnosis) that breast cancer could occur in men. ${ }^{27}$ Raising this issue may be challenging, particularly as resources promoting breast awareness are mainly aimed at women..$^{28}$ Our hospital Patient and Public Involvement group highlighted some of the issues men might face during and after receiving a breast cancer diagnosis, for example being the only man in the waiting room for appointments.

Patients may be unaware that cancer-predisposing $B R C A$ variants can be passed on by men

Harry is a 55 year old man whom you see regularly for diabetes management. At the end of an appointment discussing his blood sugars, he mentions that his sister in Australia recently told him that she "has BRCA" and that he should get tested. You ask how he feels about this, and he says he can't see the point because "isn't BRCA a female thing?" Harry has two daughters in their 30 .

Because cancer-predisposing BRCA1 and $B R C A 2$ variants are notorious for increasing lifetime risk of breast and ovarian cancer in women, there is a common misperception that cancer-predisposing $B R C A$ variants themselves only occur in women. ${ }^{29}$ Cancer-predisposing $B R C A$ variants are just as common in men as they are in women but are less likely to be detected because they are less likely to cause a cancer that prompts genetic testing.

Explain that men and women inherit these genetic variants in the same way: that any child of a parent with a cancer-predisposing $B R C A$ variant has a 50:50 chance of inheriting that variant. This would include Harry's children if Harry also has the BRCA variant identified in his sister.

For some men, concern for the health of existing or potential daughters or granddaughters is a key motivation for seeking $B R C A$ testing. ${ }^{2930}$ In contrast, sometimes men are reluctant to have $B R C A$ testing because they are concerned about potentially having passed a cancer-predisposing BRCA variant on to their children. They might prefer not to know than to have this possibility confirmed. It may help to remind them that the cancer-predisposing $B R C A$ variant has likely been in their family for generations and whether they inherited or passed it on is outside their control. However, being tested for it might guide medical care for them and their children, for example by informing choices about cancer screening (fig 2). 


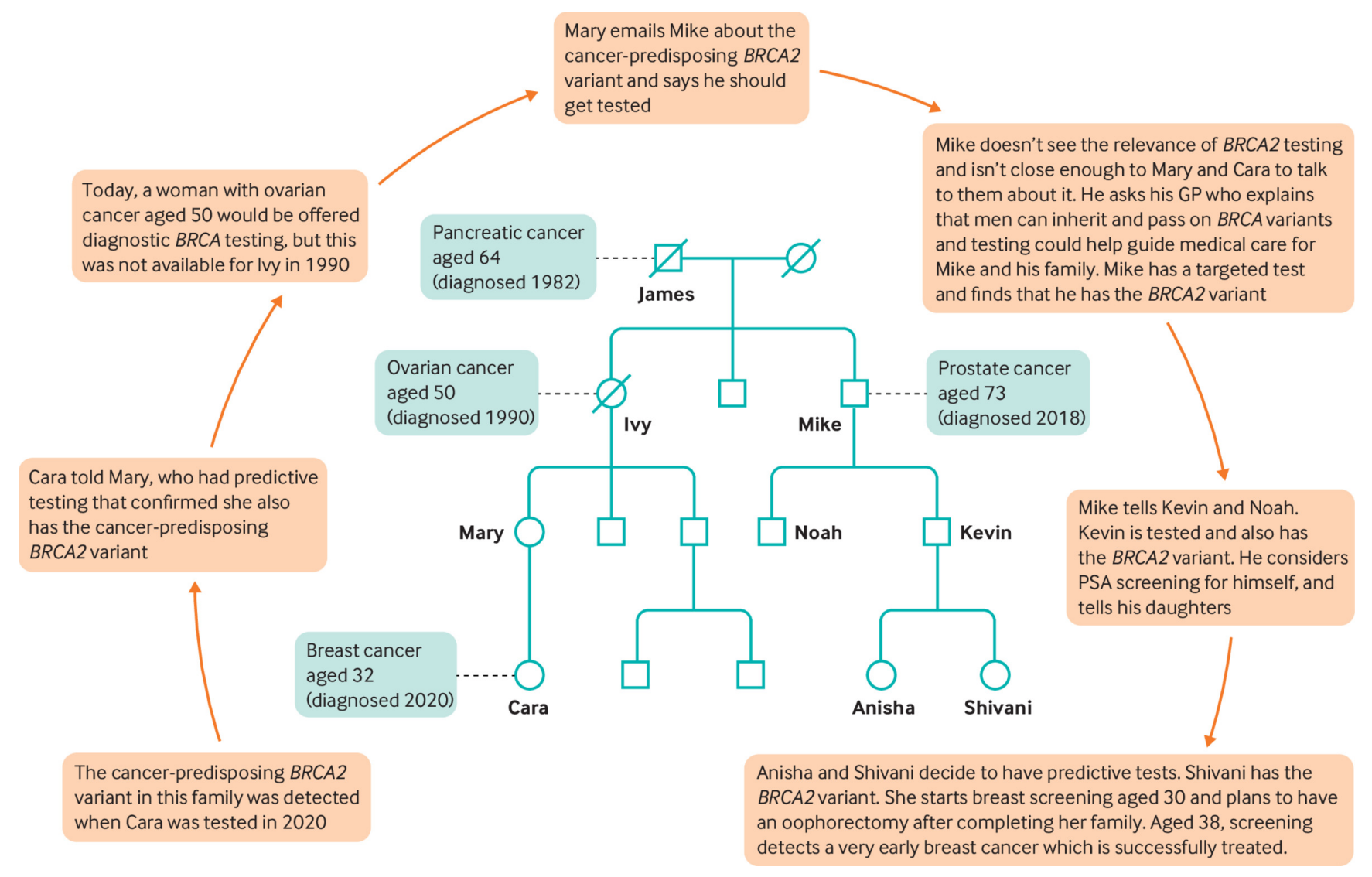

Fig 2 | A family learning about $B R C A$

Occasionally, men decide that they do not wish to be tested for a cancer-predisposing $B R C A$ variant found in their family. It would still be appropriate and important to refer their adult, first degree relatives (eg, siblings or children) to clinical genetics specialists if they wish, even if testing them might (indirectly) reveal that the man has the cancer-predisposing $B R C A$ variant. Clinical genetic services are skilled at counselling patients through difficult psychosocial and ethical issues such as this scenario (box 2).

\section{Box 2: Ethical issues}

Identifying a cancer-predisposing BRCA variant may raise various ethical issues, for example:

\section{Sharing genetic information within families}

Family members may benefit from testing for the cancer-predisposing $B R C A$ variant. What if the person in whom it was identified finds it difficult to tell their family about the variant, or chooses not to? Health professionals may sometimes need to balance the competing tensions of patient confidentiality with the interests others have in knowing about their risks.

\section{Decisions around termination of pregnancy owing to adult onset} conditions

People with cancer-predisposing $B R C A$ variants have a substantially increased risk of developing certain cancers, but these are adult-onset and some people with such variants will never develop cancer.

\section{Shifting understanding of what particular genetic variants mean}

Classifying $B R C A$ variants as benign or cancer-predisposing is technically challenging. Over time, new evidence may shift our understanding of what a particular variant means (eg, it may become clear that a variant thought to predispose to cancer is actually benign, or vice versa). How should clinicians respond when this happens?

\section{Men with cancer-predisposing $B R C A$ variants have an increased risk of developing pancreatic cancer}

Simon is a 50 year old man who had a test two years ago via a genetics clinic for the cancer-predisposing BRCA2 variant identified in his sister. Simon was also found to have the BRCA2 variant and discussed his increased risk of prostate and pancreatic cancer with a genetic counsellor at the time. Last week Simon's friend was diagnosed with pancreatic cancer. Simon is now more concerned about his risk of pancreatic cancer and books an appointment to discuss it with you.

People with cancer-predisposing $B R C A$ variants have an increased risk of developing pancreatic cancer: a retrospective cohort analysis of a high-risk breast cancer family registry from the US, Canada, and Australia estimated that pancreatic cancer risk is five to six times higher than population risk for people with cancer-predisposing $B R C A 2$ variants and around four times higher for people with cancer-predisposing $B R C A 1$ variants. ${ }^{5}$ Unfortunately, pancreatic cancer is challenging to screen for and is often advanced by the time symptoms develop. Guidance from the National Institute for Health and Care Excellence (2018) on pancreatic cancer recommended that people with cancer-predisposing $B R C A$ variants and one or more first degree relatives with pancreatic cancer have surveillance (magnetic resonance imaging/magnetic resonance cholangiopancreatography or endoscopic ultrasound), ${ }^{31}$ but this was challenged by the UK Cancer Genetics Group as being premature.$^{32}$ Instead, the group recommended that pancreatic cancer screening should only be offered within the context of research studies such as EUROPAC. ${ }^{33}$ In practice, pancreatic cancer screening is generally considered on a case-by-case basis for people with cancer-predisposing $B R C A$ variants if they also have a family 
history of pancreatic cancer. Screening as part of a research study might be a possibility for Simon-your local genetics service may be able to signpost towards this.

Around $6-7 \%$ of people with metastatic pancreatic cancer have a germline cancer-predisposing $B R C A$ variant. ${ }^{34}$ Increasingly, people with pancreatic cancer are offered diagnostic $B R C A$ testing to inform treatment plans. Cancer cells with cancer-predisposing $B R C A$ variants already have impaired DNA repair and are heavily reliant on DNA repair pathways involving PARP, so are particularly vulnerable to PARP inhibitors. PARP inhibitors may be a treatment option for people with a germline cancer-predisposing $B R C A$ variant who have breast, ovarian, pancreatic, or prostate cancer (often in the context of clinical trials). 35

\section{Education into practice}

- Do you ask about paternal as well as maternal family history when assessing a patient's risk of breast cancer?

- What questions might you ask if a man tells you he doesn't want testing for the cancer-predisposing BRCA variant found in his family?

- When did you last ask a man about his family history of breast and ovarian cancer?

\section{How patients were involved in the creation of this article}

We spoke with men from the Patient and Public Involvement group at University Hospitals Southampton NHS Foundation Trust to discuss what prior knowledge men might have about cancer-predisposing $B R C A$ variants, what information they might want to know after finding that they had a cancer-predisposing BRCA variant, and what terminology they might prefer when talking about genetic variants. These discussions particularly influenced the case study section, the discussion about expectations in "How would a man find out that he has a cancer-predisposing BRCA variant?," and the box "Describing genetic variants."

\section{How this article was made}

We developed fictitious cases to illustrate common issues that may arise for men with cancer-predisposing BRCA variants, based on scenarios encountered by our regional genetics department.

We used PubMed and author research paper archives to search for information about cancer risks and medical care for men with cancer-predisposing $B R C A$ variants. We aimed to quote cancer risks established by large prospective cohort studies. Medical care of men with cancer-predisposing $B R C A$ variants is under-researched and often guidelines are lacking-in these cases, we have drawn on our experience within a UK regional genetics department to aim to reflect UK standard practice.

Competing interests: We have read and understood the $B M$ / policy on declaration of interests and declare the following interests: none.

Contributorship and guarantor: RH drafted the article, PP edited the draft, advised on selection of evidence and created figure 1. JH edited the draft from her perspective as a GP, AL developed the article, facilitated patient and public input and edited the draft as a whole. AL is the guarantor.

Acknowledgments: We thank Gillian Crawford for her clinical insights and support with arranging patient and public involvement for this article. Thanks to the National Institute for Health Research Southampton Biomedical Research Centre and Clinical Research Facility supported Patient and Public Involvement group at the University Hospitals Southampton NHS Foundation Trust and Barney Jones and Michael Bahrami-Hessari for their input on this article.

The cases in this article are fictitious and therefore no patient consent was needed.

Funding: Rachel Horton's work is funded by a Wellcome Trust Research Award for Health Professionals in Humanities and Social Science 218092/Z/19/Z. Anneke Lucassen's work is supported by funding from a Wellcome Trust collaborative award 208053/Z/17/Z.
Maxwell KN, Domchek SM, Nathanson KL, Robson ME. Population frequency of germline BRCA1/2 mutations. J Clin Oncol 2016;34:4183-5. doi: 10.1200/JC0.2016.67.0554 pmid: 27551127

2 Dorling L, Carvalho S, Allen J, etalBreast Cancer Association Consortium. Breast cancer risk genes-association analysis in more than 113000 women. N Engl J Med 2021;384:428-39. doi: 10.1056/NEJMoa1913948 pmid: 33471991

3 Burke W, Culver J, Pinsky L, etal. Genetic assessment of breast cancer risk in primary care practice. Am J Med Genet A 2009;149A:349-56. doi: 10.1002/ajmg.a.32643 pmid: 19208375

4 Gumaste PV, Penn LA, Cymerman RM, Kirchhoff T, Polsky D, McLellan B. Skin cancer risk in BRCA1/2 mutation carriers. Br J Dermatol 2015;172:1498-506.

doi: 10.1111/bjd.13626 pmid: 25524463

Mocci E, Milne RL, Méndez-Villamil EY, etal. Risk of pancreatic cancer in breast cancer families from the breast cancer family registry. Cancer Epidemiol Biomarkers Prev 2013;22:803-11. doi: 10.1158/1055-9965.EPI-12-0195 pmid: 23456555

6 Kuchenbaecker KB, Hopper JL, Barnes DR, etalBRCA1 and BRCA2 Cohort Consortium. Risks of breast, ovarian, and contralateral breast cancer for BRCA1 and BRCA2 mutation carriers. JAMA 2017;317:2402-16. doi: 10.1001/jama.2017.7112 pmid: 28632866

7 Nyberg T, Frost D, Barrowdale D, etal. Prostate cancer risks for male BRCA1 and BRCA2 mutation carriers: a prospective cohort study. Eur Urol 2020;77:24-35.

doi: 10.1016/j.eururo.2019.08.025 pmid: 31495749

8 van Asperen CJ, Brohet RM, Meijers-Heijboer EJ, etalNetherlands Collaborative Group on Hereditary Breast Cancer (HEBON). Cancer risks in BRCA2 families: estimates for sites other than breast and ovary. J Med Genet 2005;42:711-9. doi: 10.1136/jmg.2004.028829 pmid: 16141007

9 Tai YC, Domchek S, Parmigiani G, Chen S. Breast cancer risk among male BRCA1 and BRCA2 mutation carriers. J Natl Cancer Inst2007;99:1811-4. doi: 10.1093/jnci/djm203 pmid: 18042939

10 Kuchenbaecker KB, McGuffog L, Barrowdale D, etal. Evaluation of polygenic risk scores for breast and ovarian cancer risk prediction in BRCA1 and BRCA2 mutation carriers. J Natl Cancer Inst 2017;109:djw302. doi: 10.1093/jnci/djw302 pmid: 28376175

11 BRCA Challenge Project. BRCA Exchange https://brcaexchange.org/

12 Mighton C, Charames GS, Wang M, etal. Variant classification changes over time in BRCA1 and BRCA2. Genet Med 2019;21:2248-54. doi: 10.1038/s41436-019-0493-2 pmid: 30971832

13 National Institute for Health and Care Excellence. Familial breast cancer: classification, care and managing breast cancer and related risks in people with a family history of breast cancer. (CG164) 2017. nice.org.uk/guidance/cg164

14 National Genomic Test Directory. Testing Criteria for Rare and Inherited Disease. R208 Inherited breast cancer and ovarian cancer. https://www.england.nhs.uk/wp-content/uploads/2018/08/Rareand-Inherited-Disease-Eligibility-Criteria-November-2020-21.pdf2020

15 Oh M, Alkhushaym N, Fallatah S, etal. The association of BRCA1 and BRCA2 mutations with prostate cancer risk, frequency, and mortality: A meta-analysis. Prostate 2019;79:880-95. doi: 10.1002/pros.23795 pmid: 30900310

16100000 Genomes Project. Additional findings https://www.genomicsengland.co.uk/informationfor-participants/additional-findings/

17 Horton R, Crawford G, Freeman L, Fenwick A, Wright CF, Lucassen A. Direct-to-consumer genetic testing. BMJ 2019;367:15688. doi: 10.1136/bmj.l5688 pmid: 31619392

18 Castro E, Goh C, Olmos D, etal. Germline BRCA mutations are associated with higher risk of nodal involvement, distant metastasis, and poor survival outcomes in prostate cancer. J Clin Oncol 2013;31:1748-57. doi: 10.1200/JCO.2012.43.1882 pmid: 23569316

19 Thompson D, Easton DF, Consortium BCLBreast Cancer Linkage Consortium. Cancer Incidence in BRCA1 mutation carriers. J Natl Cancer Inst 2002;94:1358-65.

doi: 10.1093/jnci/94.18.1358 pmid: 12237281

20 Leongamornlert D, Mahmud N, Tymrakiewicz M, etalUKGPCS Collaborators. Germline BRCA1 mutations increase prostate cancer risk. Br J Cancer 2012;106:1697-701. doi: 10.1038/bjc.2012.146 pmid: 22516946

21 Page EC, Bancroft EK, Brook MN, etallMPACT Study Collaborators. Interim results from the IMPACT study: evidence for prostate-specific antigen screening in BRCA2 mutation carriers. Eur Urol 2019;76:831-42. doi: 10.1016/j.eururo.2019.08.019 pmid: 31537406

22 European Association of Urology. EAU Guidelines: prostate cancer. Edn. presented at the EAU Annual Congress Milan 2021. https://uroweb.org/guideline/prostate-cancer/\#5

23 Nofal MN, Yousef AJ. The diagnosis of male breast cancer. Neth J Med 2019;77:356-9.pmid: 31880271

24 Gargiulo P, Pensabene M, Milano M, etal. Long-term survival and BRCA status in male breast cancer: a retrospective single-center analysis. BMC Cancer 2016;16:375. doi: 10.1186/s12885-016-2414-y pmid: 27377827

25 Ottini L, Silvestri V, Rizzolo P, etal. Clinical and pathologic characteristics of BRCA-positive and BRCA-negative male breast cancer patients: results from a collaborative multicenter study in Italy. Breast Cancer Res Treat2012;134:411-8. doi: 10.1007/s10549-012-2062-0 pmid: 22527108

26 Woods RW, Salkowski LR, Elezaby M, Burnside ES, Strigel RM, Fowler AM. Image-based screening for men at high risk for breast cancer: Benefits and drawbacks. Clin Imaging 2020;60:84-9. doi: 10.1016/j.clinimag.2019.11.005 pmid: 31864206

27 Co M, Lee A, Kwong A. Delayed presentation, diagnosis, and psychosocial aspects of male breast cancer. Cancer Med 2020;9:3305-9. doi: 10.1002/cam4.2953 pmid: 32167660

28 NHS. How should I check my breasts? 2021. https://www.nhs.uk/common-health-questions/womens-health/how-should-i-check-my-breasts/2021 
29 Hallowell N, Arden-Jones A, Eeles R, etal. Guilt, blame and responsibility: men's understanding of their role in the transmission of BRCA1/2 mutations within their family. Sociol Health Illn 2006;28:969-88. doi: 10.1111/j.1467-9566.2006.00515.x pmid: 17163862

30 Rauscher EA, Dean M, Campbell-Salome G, Barbour JB. "How do we rally around the one who was positive?" Familial uncertainty management in the context of men managing BRCA-related cancer risks. Soc Sci Med 2019;242:112592. doi: 10.1016/j.socscimed.2019.112592 pmid: 31629161

31 National Institute for Health and Care Excellence. NG85: Pancreatic cancer in adults: diagnosis and management. https://www.nice.org.uk/guidance/ng852018

32 UK Cancer Genetics Group. UKCGG Response to NICE Guideline NG85: Pancreatic Cancer. https://www.ukcgg.org/news/ukcgg-response-to-nice-guideline-ng85-pancreatic-cancer/2018

33 EUROPAC. https://www.lctu.org.uk/LCTU_NET/Frontend/?Data=W1tiRzlqWVd4bF1dW09RPT1d2021

34 Golan T, Kindler HL, Park JO, etal. Geographic and ethnic heterogeneity in the BRCA1/2 pre-screening population for the randomized phase III POLO study of olaparib maintenance in metastatic pancreatic cancer (mPC). J Clin Oncol

2018;36(suppl):4115doi: 10.1200/JCO.2018.36.15_suppl.4115

35 Schettini F, Giudici F, Bernocchi O, etal. Poly (ADP-ribose) polymerase inhibitors in solid tumours: Systematic review and meta-analysis. Eur J Cancer 2021;149:134-52 doi: 10.1016/j.ejca.2021.02.035 pmid: 33862496

36 Hodgson J, Hughes E, Lambert C. "SLANG" - sensitive language and the new genetics-an exploratory study. J Genet Couns 2005;14:415-21. doi: 10.1007/s10897-005-5886-5 pmid: 16502338

37 Wakefield CE, Meiser B, Homewood J, Barlow-Stewart K, Tucker K. A comparison of community, clinician, and patient preferences for naming a cancer-related mutation. Clin Genet2007:71:140-7. doi: 10.1111/j.1399-0004.2007.00754.x pmid: 17250662 